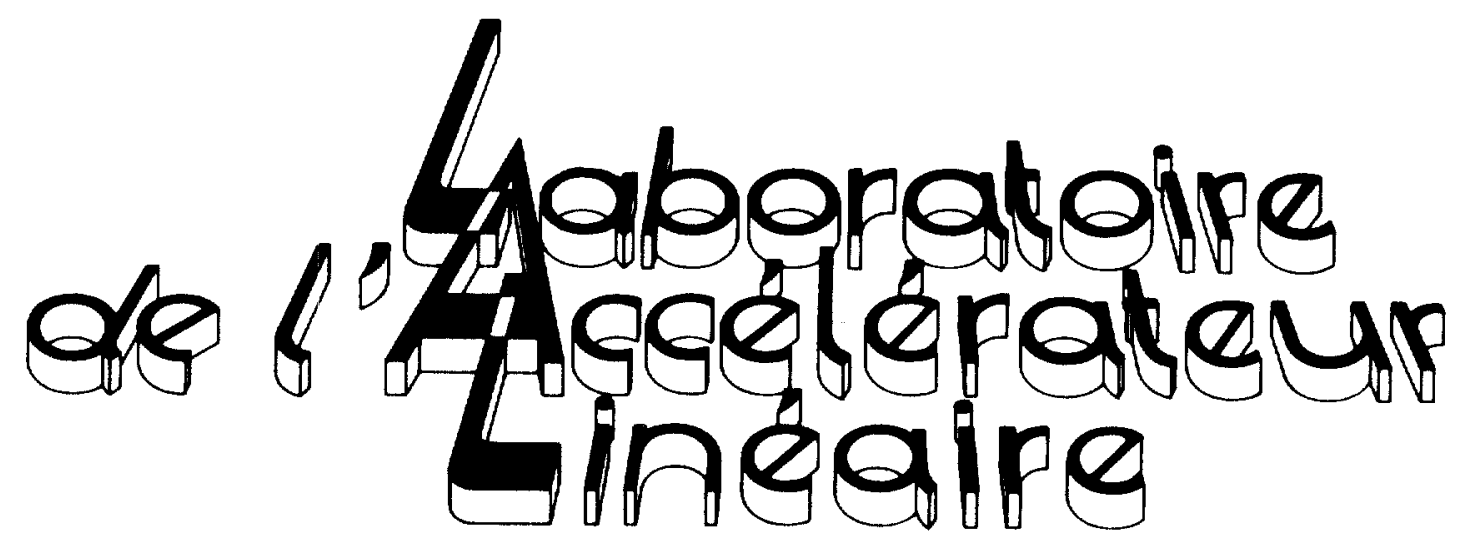

Neutrino Oscillations from Pion Decay in Flight

\author{
J.-E. Campagne \\ Laboratoire de L'Accélérateur Linéaire \\ IN2P3-CNRS et Université de Paris-Sud, F-91405 Orsay
}

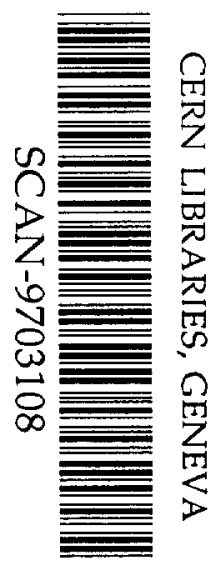

Swg773

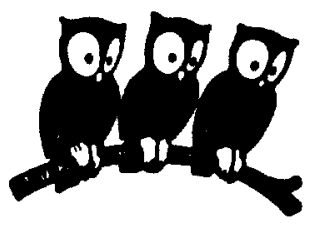

Institut National de Physique Nucleaire et de Physique des Particules 
LAL $97-03$

February 1997

\title{
Neutrino Oscillations from Pion Decay in Flight
}

\author{
J.-E. Campagne \\ Laboratoire de L'Accélérateur Linéaire \\ IN2P3-CNRS et Université de Paris-Sud, F-91405 Orsay
}

Submitted to Physics Letters B 


\title{
Neutrino Oscillations from Pion Decay in Flight
}

\author{
J.E. Campagne \\ Laboratoire de l'Accélérateur Linéaire, \\ IN2P3-CNRS et Université de Paris-Sud, 91405 Orsay, France
}

\begin{abstract}
Presented is a field theory treatment of neutrino flavor oscillations in the process $p \stackrel{T}{\longrightarrow}\left(X^{\prime}\right)+\pi^{+} \rightarrow\left(\mu^{+}\right)+\nu \stackrel{D}{\longrightarrow} l^{\mp}+(X)$, where $\mathrm{T}$ and $D$ stand for the target and detector, respectively. Without arbitrary wave-packets, it is shown that with the introduction of the experimental operating parameters to constraint the phase space the different limitations of the "classical" derivation of the oscillating amplitude are generated. The conditions of the NOMAD experiment performed at CERN are used as a guide line.
\end{abstract}

\section{Introduction}

If Dirac or Majorana neutrinos have non degenerate masses and mix together, it is well known that flavor oscillations can take place. In this case the flavor eigenstates $\nu_{l}$ and the mass eigenstates $\nu_{j}$ are related by the unitary matrix $U$

$$
\nu_{l}=\sum_{j} U_{\mu j}^{\dagger} \nu_{j}
$$

where $j$ runs from 1 up to the total number of neutrino families. In the case of two families the mixing matrix $U$ can be parametrized as

$$
U=\left(\begin{array}{cc}
\cos \theta & \sin \theta e^{i \delta} \\
-\sin \theta e^{-i \delta} & \cos \theta
\end{array}\right)
$$

where $\delta$ is a phase which can be set to zero for Dirac neutrinos, or when $C P$ is conserved for Majorana neutrinos. The oscillation probability formula is then simply [1] 


$$
\begin{aligned}
\tilde{P}\left(\nu_{\mu} \rightarrow \nu_{l}\right) & =\int d z d E_{\nu} f(z) S\left(E_{\nu}\right)\left|\sum_{j=1}^{2} U_{j l}^{*} U_{j \mu} e^{-i \frac{m_{j}^{2}}{2 E_{\nu}} z}\right|^{2} \\
& =\int d z d E_{\nu} f(z) S\left(E_{\nu}\right) \sin ^{2} 2 \theta \sin ^{2}\left(1.27 z[\mathrm{~m}] \frac{\Delta m^{2}\left[\mathrm{eV}^{2}\right]}{E_{\nu}[\mathrm{MeV}]}\right)
\end{aligned}
$$

where the spatial extensions of the source and of the detector are taken into account via the distribution $f(z)$ of the neutrino path length $z$, while the function $S\left(E_{\nu}\right)$ is the energy spectrum of the incident neutrinos.

It was realized fifteen years ago [2], that the "classical" derivation of the neutrino flavor oscillation formula (3) may be questionable. Recently, various treatments of the problem have been performed: time dependent perturbation theory in quantum mechanics [3], quantum field theory with wave-packet treatments for the external particles involved in the process [4], and treatments without wave-packets [5]. All the authors of these approaches focus their attention on the production area (radioactive nuclei, unstable particles) of the mediated virtual neutrinos as well as on the detection area which are both localized in space and separated from each other by a macroscopic distance.

In this paper the field theory approach is applied to the process of Fig. 1. A proton hits a target, at $z_{T}=0$, and produces a charged pion. Here a $\pi^{+}$pion is used, but the treatment may be applied equally well to $\pi^{-}$pion. The $\pi^{+}$ inside a vacuum tunnel decays into a $\mu^{+}$, called the primary muon, and a $\nu_{\mu}$ neutrino which in turn is detected at $z_{D}$ via charged lepton identification $(l)$. The pions are stopped in a beam dump, and the muons which get through the beam dump are deflected by a bending magnet. The external particles are described by plane waves, and the detector is assumed to perform perfect measurements; this narrows the discussion on the intrinsic limitations of the classical derivation.

This paper is organized as follows. In Sec. 2, the fact that the oscillations do not occur when four momenta are strictly conserved is reviewed. Then, the size of the target and detector as well as the length of the pion decay tunnel are introduced in Sec. 3 to make clear the role of the uncertainty principle in the momentum spread. These finite size effects as well as the pion decay width lead to the breakdown of the classical treatment of neutrino oscillations, for which the domain of validity is described in Sec. 4. The amplitude of the complete lepton number conserving process (i.e. in which the charge of the lepton $l$ is the opposite of the charge of the primary muon) is presented in Sec. 5, while the lepton number violating process in which the charge of the lepton $l$ is the same as the charge of the primary muon is presented in Sec. 6. 


\section{The "paradox" of the oscillating process}

First consider the oscillation process $\nu_{\mu} \rightarrow \nu_{l}$ as shown in Fig. 1. The $\pi^{+}$is produced by the strong interaction of a proton on a target $(T)$. Then it decays into a muon neutrino and a $\mu^{+}$. In the detector $(D)$, the charged current weak interaction of the $\nu_{\mu}$ neutrino is observed by identifying a $\mu^{-}$, but one expects to identify a $l^{-}$in case of neutrino flavor oscillations. This process is hereafter called a $\pi^{+} \mu^{+} l^{-}$-coincidence. In the following sections, Dirac neutrinos are assumed if not otherwise mentioned.

To the lowest order, the amplitude of the complete process $p \stackrel{T}{\longrightarrow}\left(X^{\prime}\right)+\pi^{+} \rightarrow$ $\mu^{+}+\nu_{\mu} \stackrel{D}{\longrightarrow} l^{-}+(X)$ with $l=\mu$ or $l \neq \mu$ (X and $X^{\prime}$ being outgoing hadrons) is equal to

$$
\mathcal{A}^{+-}=\frac{(-i)^{3}}{3 !} \int d^{4} x_{1} d^{4} x_{2} d^{4} x_{3}\left\langle f\left|T \mathcal{L}^{\nu l}\left(x_{1}\right) \mathcal{L}^{\pi \mu \nu}\left(x_{2}\right) \mathcal{L}^{p \pi}\left(x_{3}\right)\right| i\right\rangle,
$$

where $|f\rangle \equiv\left|X^{\prime} \mu^{+} l^{-} X\right\rangle$ and $|i\rangle \equiv|p T D\rangle$. The neutrino charged weak interaction at $x_{1}$, the pion leptonic decay at $x_{2}$ and the proton interaction in the target at $x_{3}$ are described by the following Lagrangians

$$
\begin{aligned}
\mathcal{L}^{\nu l}\left(x_{1}\right) & \equiv i \frac{G_{F}}{\sqrt{2}}\left[\bar{l}\left(x_{1}\right) \omega^{\rho} \nu_{l}\left(x_{1}\right) \times H_{\rho}\left(x_{1}\right)+h . c\right] \\
\mathcal{L}^{\pi \mu \nu}\left(x_{2}\right) & \equiv i f_{\pi} \frac{G_{F}}{\sqrt{2}} \cos \theta_{c}\left[\partial_{\lambda} \phi_{\pi}\left(x_{2}\right) \times \overline{\nu_{\mu}}\left(x_{2}\right) \omega^{\lambda} \mu\left(x_{2}\right)+h . c\right] \\
\mathcal{L}^{p \pi}\left(x_{3}\right) & \equiv i G\left[H^{\prime}\left(x_{3}\right) \times \phi_{\pi}^{\dagger}\left(x_{3}\right)+h . c\right]
\end{aligned}
$$

where the notation $\omega^{\mu} \equiv \gamma^{\mu}\left(1-\gamma^{5}\right)$ has been used. The hadronic currents involved in the target and in the detector are noted by $H^{\prime}$ and $H$, respectively.

The non vanishing contribution to the amplitude (4) is proportional to

$$
\begin{aligned}
& T \mathcal{L}^{\nu l}\left(x_{1}\right) \mathcal{L}^{\pi \mu \nu}\left(x_{2}\right) \mathcal{L}^{p \pi}\left(x_{3}\right) \propto \omega_{\zeta \zeta^{\prime}}^{\rho} \omega_{\eta^{\prime}}^{\lambda}\left\langle l^{-}\left|: \bar{l}_{\zeta}\left(x_{1}\right):\right| 0\right\rangle\left\langle\mu^{+}\left|: \mu_{\eta^{\prime}}\left(x_{2}\right):\right| 0\right\rangle \\
& \times\left\langle X\left|: H_{\rho}\left(x_{1}\right):\right| D\right\rangle\left\langle X^{\prime}\left|: H^{\prime}\left(x_{3}\right):\right| p T\right\rangle \\
& \times\left\langle 0\left|T \nu_{l_{\zeta^{\prime}}}\left(x_{1}\right) \bar{\nu}_{\mu_{\eta}}\left(x_{2}\right)\right| 0\right\rangle\left\langle 0\left|T \phi_{\pi}^{\dagger}\left(x_{3}\right) \partial_{\lambda} \phi_{\pi}\left(x_{2}\right)\right| 0\right\rangle,
\end{aligned}
$$

which exhibits the propagators of the pion and the neutrino. For the propagator of the pion, the Breit-Wigner approximation is used

$$
\left\langle 0\left|T \phi_{\pi}^{\dagger}\left(x_{3}\right) \phi_{\pi}\left(x_{2}\right)\right| 0\right\rangle=i \int \frac{d^{4} k}{(2 \pi)^{4}} \frac{e^{i p \cdot\left(x_{3}-x_{2}\right)}}{k^{2}-m_{\pi}^{2}+i \Gamma_{\pi} m_{\pi}},
$$

where $m_{\pi}$ and $\Gamma_{\pi}$ are the mass and width of the pion. The flavor changing neutrino propagator is equal to 


$$
\begin{aligned}
\left\langle 0\left|T \nu_{\zeta^{\prime}}\left(x_{1}\right) \overline{\nu_{\mu^{\prime} \eta}}\left(x_{2}\right)\right| 0\right\rangle & =i \sum_{j} U_{j l}^{*} U_{j \mu} S^{F}\left(x_{1}-x_{2} ; m_{j}\right)_{\zeta^{\prime} \eta} \\
& =i \sum_{j} U_{j l}^{*} U_{j \mu} \int \frac{d^{4} q}{(2 \pi)^{4}} \frac{e^{-i q \cdot\left(x_{1}-x_{2}\right)}}{\left(q-m_{j}+i \epsilon\right)_{\zeta^{\prime} \eta}} .
\end{aligned}
$$

This leads to the following expression of the amplitude $\mathcal{A}^{+-}$

$$
\begin{aligned}
& \mathcal{A}^{+-}=i^{2} \frac{G_{F}^{2} f_{\pi} \cos \theta_{c} G}{3 ! 2(2 \pi)^{8}} \omega_{\zeta \zeta^{\prime}}^{\rho} \omega_{\eta \eta^{\prime}}^{\lambda} \bar{u}_{\zeta}^{l} v_{\eta^{\prime}}^{\mu} H_{\rho}^{X D} H^{X^{\prime} p T} \\
& \times \iiint d^{4} x_{1} d^{1} x_{2} d^{4} x_{3} e^{i\left(p_{l}+p_{X}-p_{D}\right) \cdot x_{1}} e^{i p_{\mu^{\prime}} \cdot x_{2}} e^{i\left(p_{X^{\prime}}-p_{p}-p_{T}\right) \cdot x_{3}} \\
& \times\left(\int d^{4} k \frac{k_{\lambda} e^{i k^{\prime}\left(x_{3}-x_{2}\right)}}{k^{2}-m_{\pi}^{2}+i \Gamma_{\pi} m_{\pi}}\right)\left(\sum_{j} U_{j l}^{*} U_{j \mu} \int d^{4} q \frac{e^{-i q \cdot\left(x_{1}-x_{2}\right)}}{\left(\phi-m_{j}+i \epsilon\right)_{\zeta^{\prime} \eta}}\right),
\end{aligned}
$$

where the space invariant parts of the hadronic currents have been factored out.

If at this stage, one performs the integration over the $\left(x_{1}, x_{2}, x_{3}\right)$ variables, all the exponential terms are transformed into delta functions and the energymomentum conservation at each vertex occurs strictly. No oscillations can occur under these conditions; this is the essence of the "paradox". It is noteworthy in this case that the four momenta of the pion and neutrino are equal to

$$
k_{\pi}=p_{p}-p_{X^{\prime}}, \quad q_{\nu}=k_{\pi}-p_{\mu}=p_{l}+p_{X},
$$

where $p_{D}$ and $p_{T}$ have been set to zero for simplicity.

\section{The finite size effects}

To recover the oscillating pattern, one must take into account, not only the length of the proton target $T\left(\sigma_{T}\right)$ where the pion is produced but also the length of the detector $D\left(\sigma_{D}\right)$ where the production of the lepton $l$ is detected at a distance $z_{D}$ from $T[3-5]$. Additionally, the length of the pion decay tunnel $\left(l_{\pi}\right)$ is limited by the proton target and the beam dump. In the following calculation, the experimental conditions are those [see Table 1] of the NOMAD experiment:

$$
z_{D}>l_{\pi} \gg \sigma_{D}, \sigma_{T}
$$

The space constraints in the directions perpendicular to the beam axis are neglected for convenience, but there is no loss of generality as the events studied have a very low transverse momentum with respect to their longitudinal momentum. So, the finite longitudinal size of the experimental pieces, can be 
taken into account by using the one dimensional "gate" function $\Pi(z ; a, b)$ equal to 1 when $-a<z<b$ and 0 everywhere else. Also the notation $\Pi(z ; a) \equiv \Pi\left(z ;-\frac{a}{2}, \frac{a}{2}\right)$ is used. In this case the amplitude becomes proportional to

$$
\begin{aligned}
\mathcal{A}^{+-} & \propto \sum_{j} \iint d^{4} q d^{4} k \iiint d^{4} x_{1} d^{4} x_{2} d^{4} x_{3}\left(\frac{U_{j l}^{*} U_{j \mu}}{\left(\not-m_{j}+i \epsilon\right)_{\zeta^{\prime} \eta}}\right) \\
& \times\left(\frac{k_{\lambda}}{k^{2}-m_{\pi}^{2}+i \Gamma_{\pi} m_{\pi}}\right) e^{i\left(p_{l}+p_{X}-q\right) \cdot x_{1}} \Pi\left(z_{1}-z_{D} ; \sigma_{D}\right) \\
& \times e^{i\left(p_{X^{\prime}}-p_{p}+k\right) \cdot x_{3}} \Pi\left(z_{3} ; \sigma_{T}\right) e^{i\left(p_{\mu}+q-k\right) \cdot x_{2}} \Pi\left(z_{2}-z_{3} ; 0, l_{\pi}\right),
\end{aligned}
$$

where the interaction strength constants, the spinors, and the hadronic parts of Eq. (11) have been omitted.

The integral over the three times $\left(t_{1}, t_{2}, t_{3}\right)$ and the three transverse directions $\left(x_{1_{\perp}}, x_{2_{\perp}}, x_{3_{\perp}}\right)$ are not limited, and this leads to energy and transverse momentum conservation by means of the following delta functions

$$
\begin{aligned}
& (2 \pi)^{3} \delta\left(q^{0}-E_{\nu}\right) \delta\left(k^{0}-E_{\pi}\right) \delta\left(E_{\mu}+E_{\nu}-E_{\pi}\right) \\
\times & (2 \pi)^{6} \delta^{2}\left(q_{\perp}-q_{\nu_{\perp}}\right) \delta^{2}\left(k_{\perp}-k_{\pi_{\perp}}\right) \delta^{2}\left(p_{\mu_{\perp}}+q_{\nu_{\perp}}-k_{\pi_{\perp}}\right) .
\end{aligned}
$$

The variables $\left(E_{\nu}, E_{\pi}, q_{\nu_{\perp}}, k_{\pi_{\perp}}\right)$ are the conserved components of the four momenta introduced in Eq. (12) and are defined as

$$
\begin{aligned}
E_{\nu} & \equiv E_{l}+E_{X}, & E_{\pi} & \equiv E_{p}-E_{X^{\prime}} \\
q_{\nu_{\perp}} & \equiv p_{l_{\perp}}+p_{X_{\perp}}, & k_{\pi_{\perp}} & \equiv p_{p_{\perp}}-p_{X_{\perp}^{\prime}} .
\end{aligned}
$$

Now turn to the integral for the neutrino propagator. It yields:

$$
\begin{aligned}
\int d^{4} q \delta\left(q^{0}-E_{\nu}\right) \delta^{2}\left(q_{\perp}-q_{\nu_{\perp}}\right) \frac{\left(\not d+m_{j}\right)_{\zeta^{\prime} \eta}}{q^{2}-m_{j}^{2}+i \epsilon} e^{i z_{12} q_{z}} & \\
= & (-i \pi) \frac{\left(\phi_{\nu_{j}}+m_{j}\right)_{\zeta^{\prime} \eta}}{q_{\nu_{j z}}} e^{i q_{\nu_{z}} z_{12}},
\end{aligned}
$$

where $z_{12} \equiv z_{1}-z_{2}$, and the four momentum of the neutrino $\nu_{j}$ has been defined as

$$
q_{\nu_{j}} \equiv\left[E_{\nu}, q_{\nu_{\perp}}, q_{\nu_{j z}}\right] \quad \text { with } \quad q_{\nu_{j z}} \equiv\left(E_{\nu}^{2}-q_{\nu_{\perp}}^{2}-m_{j}^{2}\right)^{1 / 2}
$$

Similarly, the integral over the pion propagator gives

$$
\begin{aligned}
& \int d^{4} k \delta\left(k^{0}-E_{\pi}\right) \delta^{2}\left(k_{\perp}-k_{\pi_{\perp}}\right) \frac{k_{\lambda}}{k^{2}-m_{\pi}^{2}+i \Gamma_{\pi} m_{\pi}} e^{i z_{23} k_{z}} \\
& =(-i \pi) \frac{k_{\pi_{\lambda}}}{k_{\pi_{z}}} e^{i k_{\pi_{z}} z_{23}} e^{-\frac{z_{23}}{2 L_{\pi}}},
\end{aligned}
$$


where the four momentum of the pion has been defined as in Eq. (19)

$$
k_{\pi} \equiv\left[E_{\pi}, k_{\pi_{\perp}}, k_{\pi_{i}}\right] \quad \text { with } \quad k_{\pi_{z}} \equiv\left(E_{\pi}^{2}-k_{\pi_{\perp}}^{2}-m_{\pi}^{2}\right)^{1 / 2}
$$

and the decay length of the pion $L_{\pi} \equiv \frac{k_{\pi_{z}}}{\Gamma_{\pi} m_{\pi}}$ has been introduced. To derive Eqs. (18) and (20), one assumes the relations $q_{\nu_{j z}} z_{12} \gg 1, k_{\pi_{z}} z_{23} \gg 1$ and $L_{\pi} k_{\pi_{z}} \gg 1$ which are well justified in practice [see Table 1].

Then one is left with the integration over the $z_{1}, z_{2}$ and $z_{3}$ variables [see Eqs. (14), (18), (20)]

$$
\begin{aligned}
& I \equiv \iiint d z_{1} d z_{2} d z_{3} e^{i{q_{\nu} z}_{\nu_{12}} z_{12}} e^{i k_{\pi_{z}} z_{23}} e^{-\frac{z_{23}}{2 L_{\pi}}} e^{-i \overline{q_{\nu}} z_{1}} e^{-i p_{\mu_{z}} z_{2}} e^{i \overline{k_{\pi}} z_{3}} \\
& \times \Pi\left(z_{1}-z_{D} ; \sigma_{D}\right) \Pi\left(z_{2}-z_{3} ; 0, l_{\pi}\right) \Pi\left(z_{3} ; \sigma_{T}\right),
\end{aligned}
$$

where

$$
\overline{q_{l^{\prime} z}} \equiv\left(p_{l}+p_{X^{\prime}}\right)_{z} \quad \text { and } \quad \overline{k_{\pi z}} \equiv\left(p_{p}-p_{X^{\prime}}\right)_{z}
$$

are the components of the four momenta introduced in Eq. (12) which are not strictly conserved. One can then write the result, as

$$
\begin{aligned}
I= & e^{\left.i\left(q_{\nu_{j} z}-\overline{q_{\nu_{z}}}\right) z_{l}\right)} \times \pi \Delta_{1}\left[q_{\nu_{j z}}-\overline{q_{\nu}} ; \frac{2 \pi}{\sigma_{D}}\right] \times \pi \Delta_{1}\left[q_{\nu_{j z}}-\overline{k_{\pi z}}+p_{\mu_{z}} ; \frac{2 \pi}{\sigma_{T}}\right] \\
& \times \pi \Delta_{2}\left[-q_{\nu_{j z}}+k_{\pi_{z}}-p_{\mu_{z}} ; L^{-1}\left(l_{\pi}, L_{\pi}\right)\right] .
\end{aligned}
$$

The $\Delta_{1}$ function is defined as,

$$
\Delta_{1}\left[q ; \frac{2 \pi}{\sigma}\right] \equiv \frac{2}{\pi q} \sin \left(\frac{q \sigma}{2}\right)
$$

and has a well known width of the order of $\frac{2 \pi}{\sigma}$ and has the property $\Delta_{1}[q, 0]=$ $\delta(q)$. Similarly, the $\Delta_{2}$ function which has been defined as

$$
\begin{aligned}
& \Delta_{2}\left[q ; L^{-1}\left(l_{\pi}, L_{\pi}\right)\right] \equiv e^{i q \frac{l_{\pi}}{2}} e^{\frac{l_{\pi}}{4 L_{\pi}}} \frac{2}{i q-\frac{1}{2 L_{\pi}}} \\
& \quad \times\left[i \sin \left(q \frac{l_{\pi}}{2}\right) \cosh \left(\frac{l_{\pi}}{4 L_{\pi}}\right)-\cos \left(q \frac{l_{\pi}}{2}\right) \sinh \left(\frac{l_{\pi}}{4 L_{\pi}}\right)\right]
\end{aligned}
$$

and has a modulus with a resonante behaviour and width of the order of

$$
L^{-1}\left(l_{\pi}, L_{\pi}\right) \simeq \frac{\sqrt{2}}{L_{\pi}}\left[\frac{\cosh \left(\frac{l_{\pi}}{2 L_{\pi}}\right)-1}{2\left(\cosh \left(\frac{l_{\pi}}{2 l_{\pi}}\right)-1\right)-\left(\frac{l_{\pi}}{2 l_{\pi}}\right)^{2}}\right]^{1 / 2}
$$


When $L_{\pi} \gg l_{\pi}$ one obtains a limit on the $\Delta_{2}$ function in terms of the $\Delta_{1}$ function, namely

$$
\Delta_{2}\left[q ; L^{-1}\left(l_{\pi}, L_{\pi}\right)\right] \approx e^{i q \frac{l_{\pi}}{2}} \Delta_{1}\left[q ; \frac{2 \pi}{l_{\pi}}\right]
$$

with a width of the order of $\frac{2 \pi}{l_{\pi}}$, and when $L_{\pi} \ll l_{\pi}$ the width of the $\Delta_{2}$ function is of order $\frac{1}{L_{\pi}}$.

Putting the results of Eqs. (15), (18), (20), (24) together, the expression for the amplitude $\mathcal{A}^{+-}$(14) becomes

$$
\begin{aligned}
& \mathcal{A}^{+-}=\frac{G_{F}^{2} f_{\pi} \cos \theta_{c} G}{3 !} \pi^{6} \delta\left(E_{F}-E_{p}\right) \delta^{2}\left(p_{F_{\perp}}-p_{p_{\perp}}\right) \\
& \times \frac{H^{X^{\prime} p T}}{k_{\pi_{z}}} \bar{u}^{l}\left(\omega^{\rho} H_{\rho}^{X \cdot D}\right)\left[\sum_{j} U_{j l}^{*} U_{j \mu} e^{i\left(q_{\nu_{j z}}-\overline{\bar{\nu}_{\nu_{z}}}\right) z_{D}} \mathcal{F}\left(q_{\nu_{j z}}\right)\right]\left(\omega^{\lambda} k_{\pi_{\lambda}}\right) v^{\mu} .
\end{aligned}
$$

Here, the notations $E_{F} \equiv E_{\mu}+E_{l}+E_{X}+E_{X^{\prime}}$ and $p_{F_{\perp}} \equiv p_{\mu_{\perp}}+p_{l_{\perp}}+p_{X_{\perp}}+p_{X_{\perp}^{\prime}}$ have been introduced. Using the chirality property $\omega^{\rho} \omega^{\lambda}=\gamma^{\rho}\left(1-\gamma^{5}\right) \gamma^{\lambda}(1-$ $\left.\gamma^{5}\right)=0$, the "overlap" function $\mathcal{F}\left(q_{\nu_{j z}}\right)$ similar to the $g$-function of Ref. [3] is defined as

$$
\begin{aligned}
\mathcal{F}\left(q_{\nu_{z}}\right) & \equiv\left(\frac{\phi_{\nu_{j}}}{q_{\nu_{j z}}}\right) \Delta_{1}\left[q_{\nu_{j z}}-\bar{q}_{\nu_{z}} ; \frac{2 \pi}{\sigma_{D}}\right] \Delta_{1}\left[q_{\nu_{j z}}-\overline{k_{\pi z}}+p_{\mu_{z}} ; \frac{2 \pi}{\sigma_{T}}\right] \\
& \times \Delta_{2}\left[-q_{\nu_{j z}}+k_{\pi_{z}}-p_{\mu_{z}} ; L^{-1}\left(l_{\pi}, L_{\pi}\right)\right] .
\end{aligned}
$$

Equation (29) exhibits the usual oscillation pattern [Eq. (3)], if and only if the "overlap" function can be made independent of the neutrino species $j$. This constraint leads to the limitations on the classical derivation of the oscillation probability that are examined in the next section.

\section{The validity conditions}

The initial and final states are such that

$$
\left|p_{l_{z}}+p_{X_{z}}+p_{\mu_{z}}+p_{X_{z}^{\prime}}-p_{p_{z}}\right|<\min \left(\sigma_{p_{D}}, \sigma_{p_{T}}\right),
$$

where $\sigma_{p_{D}} \equiv \frac{2 \pi}{\sigma_{D}}$ and $\sigma_{p_{T}} \equiv \frac{2 \pi}{\sigma_{T}}$. This means that the global three momentum is conserved up to the error $\min \left(\sigma_{p_{D}}, \sigma_{p_{T}}\right)$ to obtain a non vanishing overlap of the two $\Delta_{1}$ functions [see Eq. (30)]. The condition (31) is only fulfilled if the neutrinos produced by the pion decays are those detected inside the detector. For example, it might be thought that a time-of-flight measurement may help in this selection, but the different neutrino species involved in the sum (29) 
participate coherently. It is the case that for a fixed pion decay length, the difference between the time of flight of two mass eigenstates $\left(\nu_{i}, \nu_{k}\right)$ is dominated by the difference of their path lengths (spread of the pion production vertex or spread of the neutrino interaction as in Eq. (22), and not by the difference in their velocities. This leads to the following constraint when neglecting the transverse momenta and assuming relativistic neutrinos

$$
z_{D} \frac{\left|q_{\nu_{i z}}-q_{\nu_{k:}}\right|}{E_{\nu}} \approx z_{D} \frac{\Delta m_{i k}^{2}}{2 E_{\nu}^{2}} \ll \min \left(\sigma_{X_{D}}, \sigma_{X_{T}}\right)
$$

or equivalently

$$
z_{D} \ll \frac{E_{\nu}}{\max \left(\sigma_{p_{D}}, \sigma_{p_{T}}\right)} L_{i k}^{o s r} \equiv L_{i k}^{c o h}
$$

where one introduces the oscillation length ( $L_{i k}^{o s c} \equiv 4 \pi E_{\nu} / \Delta m_{i k}^{2}$ ) associated with the mass eigenstates $\left(\nu_{i}, \nu_{k}\right)$, and where the coherence length $\left(L_{i k}^{\text {coh }}\right)$ introduced in Ref. [4] has been identified.

Secondly, there must be at least two mass cigenstate neutrinos $\nu_{i}$ and $\nu_{k}$ for which

$$
\Delta_{1}\left[q_{\nu_{i z}}-\bar{q}_{\nu_{z}} ; \frac{2 \pi}{\sigma_{D}}\right] \approx \Delta_{1}\left[q_{\nu_{k z}}-\bar{q}_{\nu_{z}} ; \frac{2 \pi}{\sigma_{D}}\right]
$$

and a similar condition for the second $\Delta_{1}$ function [Eq. (30)]. So, the difference between the two longitudinal momenta $q_{\nu_{i z}}$ and $q_{\nu_{k z}}$ should satisfy

$$
\left|q_{\nu_{i z}}-q_{\nu_{k z}}\right| \ll \min \left(\sigma_{p_{D}}, \sigma_{p_{1}}\right) \text { or } L_{i k}^{o s c} \gg \max \left(\sigma_{X_{D}}, \sigma_{X_{T}}\right) \text {. }
$$

It is easy to understand that if this constraint is not fulfilled, oscillation damping occurs by destructive interferences inside the target or inside the detector after performing the sum over the different neutrino species in Eq. (29).

The two neutrinos satisfying Eqs. (33) and (35) must also satisfy a third constraint originating from the $\Delta_{2}$ function. The derivation of this new constraint is similar to equation (34) and is given by

$$
L_{i k}^{o s c} \gg L\left(l_{\pi}, L_{\pi}\right) \Rightarrow\left\{\begin{array}{lll}
L_{i k}^{o s s} \gg \frac{2 \pi}{l_{\pi}} & \text { when } & l_{\pi} \ll L_{\pi} \\
L_{i k}^{\text {osc }} \gg \frac{1}{L_{\pi}} & \text { when } & l_{\pi} \gg L_{\pi} .
\end{array}\right.
$$

If the pion decay vertex is located in a region broader than the oscillation length, then the same destructive interferences mentioned above occurs. This is because the sum over different source points contributes different phases. 


\section{The oscillating amplitude}

Assuming the constraints (33), (35) and (36) are fulfilled by a subset (at least a doublet) of mass eigenstates, it is possible to write for one neutrino $\nu_{j}$

$$
q_{\nu_{j z}} \simeq{\overline{q_{\nu}}}_{z}-\frac{m_{j}^{2}}{2 E_{\nu}}
$$

if the masses and transverse momenta of the lepton $l$ and outgoing hadrons are small with respect to their longitudinal momenta. Hereafter if one assumes $L_{\pi} \gg l_{\pi}$, then the sum over neutrino species in equation (29) involving the function $\mathcal{F}$ becomes

$$
\begin{aligned}
\sum_{j} U_{j l}^{*} U_{j \mu} e^{i\left(q_{\nu_{j z}}-\overline{\bar{q}_{\nu_{z}}}\right) z_{D}} \mathcal{F}\left(q_{\nu_{j z}}\right) \\
=\frac{\overline{q_{\nu}}}{\overline{\bar{q}_{\nu}}} \frac{1}{\pi^{3}} e^{i\left(k_{\pi_{z}}-p_{\mu_{z}}-\overline{q_{\nu_{z}}}\right) \frac{l_{\pi}}{2}}\left(\sum_{j}^{\prime} U_{j l}^{*} U_{j \mu} e^{-i \frac{m_{j}^{2}}{2 E_{\nu}} \tilde{z}_{D}}\right)
\end{aligned}
$$

with $\tilde{z}_{D} \equiv z_{D}-\frac{l_{\pi}}{2}$. The change of $z_{D}$ into $\tilde{z}_{D}$ implies that the mean neutrino production point is the middle of the decay tunnel as the pion decay is uniformly distributed in the case $L_{\pi} \gg l_{\pi}$. The prime in the summation in Eq. 38 means that one only takes the subset of neutrino species that contribute with maximal amplitude to the $\mathcal{F}\left(q_{\nu_{j z}}\right)$ function. Then, the amplitude of the complete process $p \stackrel{T}{\longrightarrow}\left(X^{\prime}\right)+\pi^{+} \rightarrow \mu^{+}+\left(\nu_{\mu} \leftrightarrow \nu_{l}\right) \stackrel{D}{\longrightarrow} l^{-}+(X)$ can be written in the factored form

$$
\begin{aligned}
& \mathcal{A}^{+-}=\frac{G_{F}^{2} f_{\pi} \cos _{c}^{\theta} G}{3 !} 2 \pi^{3} \delta\left(E_{F}-E_{p}\right) \delta^{2}\left(p_{F_{\perp}}-p_{p_{\perp}}\right) \\
& \times \frac{H^{X^{\prime} p T}}{k_{\pi_{z}}} e^{i\left(k_{\pi_{z}}-p_{\mu_{z}}-\overline{q_{\nu_{z}}}\right) \frac{l_{\pi}}{2}} \\
& \times H_{\rho}^{X D}\left(\bar{u}^{l} \gamma^{\rho}\left(1-\gamma^{5}\right) \frac{\overline{q_{\nu}}}{\overline{q_{\nu}}} k_{\pi} v^{\mu}\right)\left[\sum_{j}^{\prime} U_{j l}^{*} U_{j \mu} e^{-i \frac{m_{j}^{2}}{2 \bar{E}_{\nu}} \tilde{z}_{D}}\right]
\end{aligned}
$$

The contribution of an average neutrino mass has no importance here due to the chirality structure of the interaction. Thus writing the $\bar{q}_{\nu}$ four momentum as a sum over the different spinor helicities of the product $u^{\nu} \bar{u}^{\nu}$, the above amplitude simply becomes the product of three processes: (1) the production of the pion by the interaction of the proton inside the target, (2) the weak decay of the pion into a muon and a neutrino which in turn travels as a free particle with a four momentum $\overline{q_{\nu}}$ to the detector, where it is detected by means of (3) a charged current interaction. The oscillation pattern of the 
cross section is completely determined by the factor

$$
F^{L} \equiv\left|\sum_{j=1}^{2} U_{j l}^{*} U_{j l} e^{-i \frac{m_{j}^{2}}{2 E_{\nu}} \tilde{z}_{D}}\right|^{2}=\sin ^{2} 2 \theta \sin ^{2}\left(\pi \frac{\tilde{z}_{D}}{L_{12}}\right)
$$

in the case of the two neutrino family using the expression (2) of the matrix $U$ where one recognizes the classical formula appearing in Eq. (3).

\section{The neutrino-antineutrino transition amplitude}

Now consider the $\pi^{+} \mu^{+} l^{+}$-coincidence in contrast to the $\pi^{+} \mu^{+} l^{-}$-coincidence studied in the previous sections. This process violates the total lepton number. This situation has to be considered for massive Majorana neutrinos, but in the Dirac language this process can be understood as the chain $p \stackrel{T}{\longrightarrow}\left(X^{\prime}\right)+\pi^{+} \rightarrow$ $\mu^{+}+\left(\nu_{\mu} \leftrightarrow \overline{\nu_{l}}\right) \stackrel{D}{\rightarrow} l^{+}+(X)$ [sce Fig. 1]. In other words, there is a "neutrinoantineutrino" transition.

Following the same procedure of the previous sections, the term $\bar{\nu}_{l} \bar{\omega}^{\rho} l$ of the Lagrangian, $\mathcal{L}^{\nu l}\left(x_{1}\right)[\mathrm{Eq} .(5)]$ should be studied. One has to replace the neutrino propagator, Eq. (10), by the following flavor changing Majorana neutrino propagator

$$
\begin{aligned}
\left\langle 0\left|T \overline{\nu_{l \zeta}}\left(x_{1}\right) \bar{\nu}_{\mu_{\eta}}\left(x_{2}\right)\right| 0\right\rangle & =\sum_{j k} U_{j l} U_{k \mu}\left\langle 0\left|T \bar{\nu}_{j_{\zeta}}\left(x_{1}\right) \bar{\nu}_{k_{\eta}}\left(x_{2}\right)\right| 0\right\rangle \\
& =i \sum_{j} \varphi_{j l} U_{j l}^{*} U_{j \mu}\left[C^{T} S^{F}\left(x_{1}-x_{2} ; m_{j}\right)\right]_{\zeta \eta}
\end{aligned}
$$

The invariant phase $\varphi_{j l}$ is introduced and defined by $[6,8]$

$$
\varphi_{j l} \equiv \frac{U_{j l}}{U_{j l}^{*}} \phi_{j}
$$

where $\phi_{j}$ is the creation phase factor of the Majorana neutrino $\nu_{j}$. The charge conjugation operator matrix $C=-C^{\prime}$ has the property $C \gamma^{\prime \prime T} C^{-1}=-\gamma^{\prime \prime}$.

The same validity conditions described in Sec. 4 can be derived here, so the 
amplitude of the complete process has an expression similar to formula (39):

$$
\begin{aligned}
& \mathcal{A}^{++}=\frac{G_{F}^{2} f_{\pi} \cos \theta_{c} G}{3 !} 2 \pi^{3} \delta\left(E_{F}-E_{p}\right) \delta^{2}\left(p_{F_{\perp}}-p_{p_{\perp}}\right) \\
& \times \frac{H^{X^{\prime} p T}}{k_{\pi_{z}}} e^{i\left(k_{\pi_{z}}-p_{\mu_{z}}-\overline{q_{\nu_{z}}}\right)^{\frac{l_{\pi}}{2}}} \\
& \times\left(H^{X D}\right)_{\rho}^{\dagger} \frac{v^{l T} \omega^{\rho T}}{\overline{q_{\nu_{z}}}}\left[\sum_{j}^{\prime} \varphi_{j l} U_{j l}^{*} U_{j \mu} C^{T}\left(\phi_{\nu_{j}}+m_{j}\right) e^{-i \frac{m_{j}^{2}}{2 F_{\nu}} \bar{z}_{D}}\right]\left(\omega^{\lambda} k_{\pi_{\lambda}}\right) v^{\mu} .
\end{aligned}
$$

Using the property of the $C$ matrix mentioned above $C^{T}\left(\phi_{\nu_{j}}+m_{j}\right)=\left(-q_{\nu_{j}}+\right.$ $\left.m_{j}\right)^{T} C^{T}$. This last term can be replaced by a sum over the spinor helicities of the product $\left(\bar{\nu}^{\nu_{j}}\right)^{T}\left(v^{\nu_{j}}\right)^{T} C^{T}$. Then, using a suitable representation of the spinors such that $\bar{u}=-v^{T} C^{T}$, the above amplitude $\mathcal{A}^{++}$appears as the product of three different amplitudes as it is the case for the amplitude $\mathcal{A}^{+-}$in the previous section. But here, the neutrino travelling with a four momentum $q_{\nu j}$ is an antiparticle in the Dirac language as expected from the intuitive representation of the phenomena. To rewrite Eq. (43), use the relation

$$
v^{l T}\left[\gamma^{\rho}\left(1-\gamma^{5}\right)\right]^{T} C^{T}=\bar{u}^{l} \gamma^{\rho}\left(1+\gamma^{5}\right),
$$

and the chirality property

$$
\left(1+\gamma^{5}\right)\left(\not \nu_{j}+m_{j}\right)\left(1+\gamma^{5}\right)=2 m_{j}\left(1+\gamma^{5}\right)
$$

which exhibits the Majorana mass suppression factor. This analysis yields

$$
\begin{aligned}
& \mathcal{A}^{++}=\frac{G_{F}^{2} f_{\pi} \cos \theta_{c} G_{1}}{3 !} 2 \pi^{3} \delta\left(E_{F}-E_{p}\right) \delta^{2}\left(p_{F_{\perp}}-p_{p_{\perp}}\right) \\
& \times \frac{H^{X^{\prime} p T}}{k_{\pi_{z}}} e^{i\left(k_{\pi_{z}}-p_{\mu_{z}}-\overline{q_{\nu}}\right) \frac{l_{\pi}}{2}} \\
& \times\left(H^{X D}\right)_{\rho}^{\dagger}\left(\bar{u}^{l} \gamma^{\rho}\left(1+\gamma^{5}\right) \frac{k_{\pi}}{\overline{q_{\nu}}} v^{\mu}\right)\left[\sum_{j}^{\prime} \varphi_{j l} U_{j l}^{*} U_{j \mu} m_{j} e^{-i \frac{m_{j}^{2}}{2 E_{\nu}} \tilde{z}_{D}}\right]
\end{aligned}
$$

The $F^{K}$ factor corresponding to the $F^{L}$ factor introduced for the lepton number conserving process is defined here as

$$
\begin{aligned}
F^{V} & \equiv\left|\sum_{j=1}^{2} \varphi_{j l} m_{j} U_{j l}^{*} U_{j \mu} e^{-i \frac{m_{j}^{2}}{2 E_{\nu}} \tilde{z}_{D}}\right|^{2} \\
& =\frac{1}{4} m_{2}^{2} \sin ^{2} 2 \theta\left[1+\frac{m_{1}^{2}}{m_{2}^{2}}-2 \frac{m_{1}}{m_{2}} \cos \left(2 \pi \frac{\tilde{z}_{D}}{L_{12}}+2 \delta\right)\right]
\end{aligned}
$$

The study of the phenomonological consequences of the $\left(F^{L}, F^{L}\right)$ factors in the case of the $\tau$ lepton appearance in the $\nu_{\mu} \leftrightarrow \nu_{\tau}$ oscillations has been described in Ref. $[7,8]$. 


\section{Discussion and conclusion}

The different constraints, Eqs. (33), (35) and (36) of Sec. 4 are summarized as

$$
\left.z_{D} \ll L_{i k}^{\text {coh }} \quad \text { and } \quad L_{i k}^{o s s} \gg \max \left(\sigma_{l}\right), \sigma_{T}, L\left(l_{\pi}, L_{\pi}\right)\right)
$$

Table 1 gives the parameters of the NOMAD experimental conditions [9]. One sees that the validity of the classical treatment is limited by the finite length of the pion decay tunnel. In terms of $\Delta m^{2}$ when considering the lepton number conserving processes, a breakdown occurs for $\Delta m^{2} \geq 220 \mathrm{eV}^{2}$, while the maximum in the oscillation amplitude is reached for $\Delta m^{2} \approx 45 \mathrm{eV}^{2}$ corresponding to $L_{m a x}^{o s c}=2 \tilde{z}_{D}$. So, the tau neutrino mass may be probed up to $\approx 15 \mathrm{eV}$, in the see-saw hierarchy of the clifferent neutrino species. One is reminded that the lowest $\Delta m^{2}$ that the experiment may reach with $10^{6}$ neutrino interactions is $\Delta m^{2} \approx 0.1 \mathrm{eV}^{2}$. In contrast, the LSND experiment [10] pions decay at rest, so the limitations would come from the size of the detector $(7.5 \mathrm{~m})$ and from the proton target $(0.2 \mathrm{~m})$. But, the oscillation length corresponding to the maximum amplitude of the oscillations is $60 \mathrm{~m}$ and this is large enough to allow the use of the classical treatment [see Eq. (48) with $L_{\pi} \simeq \sigma_{T}$ ].

In conclusion, the flavor oscillations of neutrinos produced by pions decaying in flight have been studied in the framework of quantum field theory following the authors of Ref. [5] without arbitrary wave-packet treatments $[2,4]$. If experimental parameters like the proton target length, the detector length, and the decay tunnel length, as well as the width of the pion are introduced to constraint the phase space, then there naturally appear the intrinsic limitations of the classical derivation of the oscillating amplitude. Under specific conditions, one finds factored expressions of the oscillating amplitudes in the case of lepton number conserving processes where a real neutrino is travelling, as well as in the case of lepton number violating processes where a real antineutrino in the Dirac language is travelling. This factorization was the starting point of Ref. [7], and is justified in practice as shown in this paper. The pion width and the decay tunnel length have been considered separately in Ref. [3] in the framework of time dependent perturbation theory in quantum mechanies. Described in this paper, is a unified way of treating these two constraints in a more general framework. Also shown is that in the case of the NOMAD experiment, the only critical parameter may be the decay tunnel length which tends to break the classical treatment, for $\Delta m^{2} \geq 220 \mathrm{cV}^{2}$. 


\section{Acknowledgements}

We greatly appreciate the fruitful discussions with S. Petcov and Y. Stern. We also wish to thanks .J. Dumarchez and F. Federspiel who have kindly provided the operating parameters of the NOMAD and LSND experiments, respectively.

\section{References}

[1] S. Bilenky and B. Pontecorvo, Phys. Rep. 41C (1978) 225.

[2] B. Kayser, Phys. Rev. D 24 (1981) 110.

[3] J. Rich, Phys. Rev. D 48 (1993) 4318.

[4] C. Giunti, C.W. Kim, J.A Lee and U.W. Lee, Phys. Rev. D 48 (1993) 4310.

[5] W. Grimus and P. Stockinger, Phys. Rev. D 54 (1996) 3414.

[6] B. Kayser, in: CP violation, Advanced Series on Directions in High Energy Physics, ed. C. Jarkslog (World Scientific, 1989) p. 334.

[7] M. Carena, B. Lampe and C.E.M. Wagner, Phys. Lett. B 317 (1993) 112.

[8] J.E. Campagne, LAL 95-23 (1995), unpublished.

[9] NOMAD experiment, to be published in Nucl. Instrum. Methods.

[10] C. Athanassopoulos et al., LA-UR-96-1327, to be published in Nucl. Instrum. Methods. 
Fig. 1. Example of processes with possible $\nu_{\mu} \rightarrow \nu_{l}$ oscillations with Dirac neutrinos $\left(l^{-}\right)$or $\nu_{\mu} \rightarrow \overline{\nu_{l}}$ oscillations with Majorana neutrinos $\left(l^{+}\right)$. The shaded box represents the beam dump.

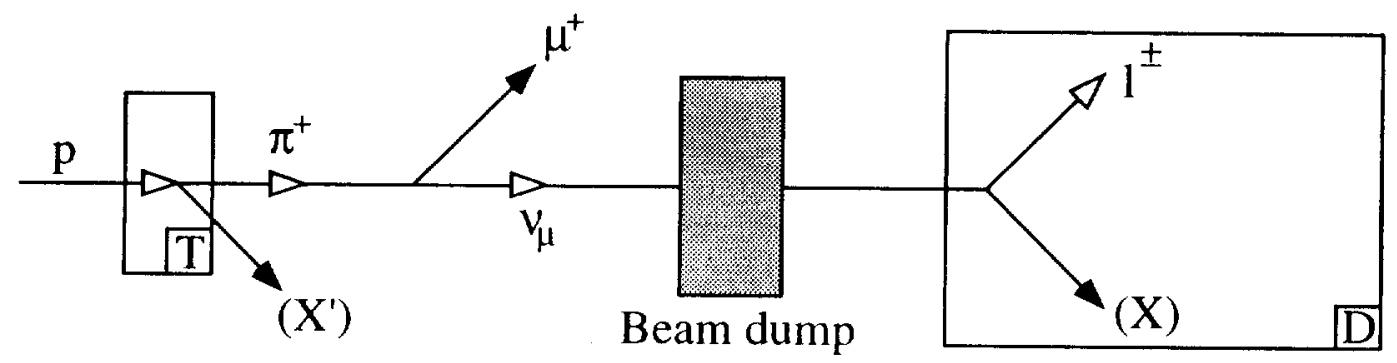

Table 1

Operating parameters of the NOMAD experiment [9]: the mean energy of the proton beam, the mean energy of the induced charged pion beam, the mean energy of the $\nu_{\mu}$ neutrino produced by the pion decays, the distance between the proton target $(T)$ and the detector $(D)$, the length of the pion decay tunnel, the decay length of the pion computed from the pion energy, the size of the proton target, the size of the detector, the coherence length (see text), the oscillation length corresponding to the maximum of the oscillation. The energies and lengths are expressed in $\mathrm{GeV}$ and in meter, respectively.

\begin{tabular}{cccccccccc}
\hline$\left\langle E_{\text {proton }}\right\rangle$ & $\left\langle E_{\pi}\right\rangle$ & $\left\langle E_{\nu_{\mu}}\right\rangle$ & $z_{D}$ & $l_{\pi}$ & $L_{\pi}$ & $\sigma_{T}$ & $\sigma_{D}$ & $L^{\text {coh }}$ & $L_{\text {max }}^{\text {osc }} \equiv 2 z_{D}-l_{\pi}$ \\
\hline 450 & 75 & 25 & 840 & 290 & 4300 & 1 & 4 & $10^{19}$ & 1390 \\
\hline
\end{tabular}

